CORRESPONDENCE.

\title{
RATES OF MORTALITY IN WEST AFRICA.
}

To the Editor of the Journal of the Institute of Actuaries.

SIR,-I have lately had occasion to investigate the experience of the Office with which I am connected, in regard to European lives residing in the Congo Free State, and have the pleasure to place the results at the disposal of the Institute of Actuaries as a supplement to the recent contribution of Mr. A. E. Sprague on this subject. The remark made by Mr. King, in discussing that paper, that considerable value would attach to statistics relating to assured lives, has induced me to submit the accompanying table.

The observations are made according to policy years, and extend over a period of six years, terminating with the anniversaries in 1896 , but no life was under observation for the special risk longer than five years. The lives, 141 in number, were almost entirely Belgians, mostly employed by the Congo Free State Government or the Congo Railway Company, and the average duration of the exposure to the climate risk was about $1 \frac{1}{2}$ years. It will be seen that the mortality in the first year of observation was appalling, particularly in the age groups 30-39 and 40-49, and if this is a fair sample of the effect of the Congo climate on Europeans, it would appear that there is no premium practically obtainable which will cover the risk of the first 
year's residence. It seems worthy of note that this experience confirms the conclusions arrived at by Mr. Sprague, (1) that the mortality in the first year of exposure to the Congo climate is far greater than in. subsequent years, and (2) that the effect of the climate is much worse on the older than on the younger lives. Probably, the Congo Railway employés were not a very eligible class of lives as regards social status, and would not exercise a very intelligent care of their health, and in the case of the Free State officials, some few took part in semi-military expeditions and were killed by natives. It will also be observed that those few lives which entered upon, also completed the fifth year of observation, there being no deaths or withdrawals, nor have any further deaths been announced since the close of the observations. This seems to indicate either a process of acclimatization, or the survival of those who were from the first best able to stand the climate.

By grouping the observations of the 3rd, 4th and 5th years, the following comparison of rates of mortality is obtained.
All Ages
$15 \cdot 38$ per-cen
1st Year.
2nd Year.
3rd-5th Years.
9.90 per-cent $\quad 7.62$ per-cent

It has not been possible to trace to any appreciable extent the effect of the Congo climate on the subsequent mortality of the lives exposed to its influence, the withdrawal from further observation being, in nearly every case, coincident with the termination of the special risk. This was probably owing to the premiums having been paid by the employers so long as the lives remained in their service, and to the lives themselves not being able to afford to take over the policies on their return. The large number of withdrawals might, however, be taken to indicate that those who returned to Europe were not, as a rule, in failing health, as it would be expected that the policies would be kept up in any such cases. There was only one case where death occurred during the existence of the policy, and after cessation of the special risk.

Possibly an experience relating to Belgian lives may be inapplicable to our own countrymen, and I believe it is generally supposed that Englishmen withstand climatic influences somewhat better than other Kuropeans. Englisbmen, however, do not seem to favour the Congro State much, and I cannot recollect a single English case being submitted to my Office. There are a few English lives residing in other parts of West Africa (e.g., Niger Protectorate, Slave Coast, Gold Coast, Sierra Leone, and on the river Gambia) on the books, but the particulars are not readily available, nor is the number sufficiently great to be of practical use. Still, I cannot call to mind a single death amongst such lives during the last nine or ten years.

Whilst I do not suggest that the figures submitted herewith are in themselves more than merely interesting, I hope that perhaps other members may find it possible to contribute the experience of their Offices, in which case the aggregate results might prove practically useful.

\section{I am, Sir,}

Your obedient Servant,

81 Cornhill,

I. K. PAGDEN.

25 March 1897. 


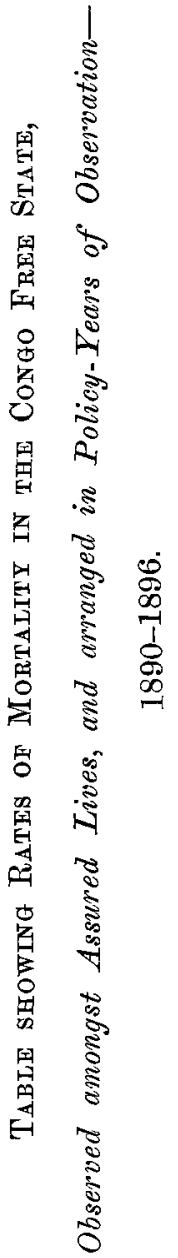

\begin{tabular}{|c|c|c|c|c|c|c|}
\hline & $\sum_{4}^{\infty}$ & 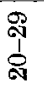 & कृ & $\begin{array}{l}9 \\
\underset{7}{1} \\
0\end{array}$ & $\underbrace{8} \underbrace{8}$ & $=\underbrace{\infty}$ \\
\hline \multirow{6}{*}{ 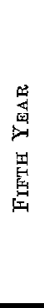 } & 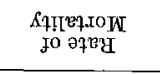 & 乌్ & $\S$ & $\vdots$ & 乌్̊ & § \\
\hline & әd!T jo s.ॅв X & -1 & $n$ & $\vdots$ & $r$ & $\sigma$ \\
\hline & Sut!s!x' & -1 & $N$ & $\vdots$ & -1 & $\sigma$ \\
\hline & вІямегрч7!М & 0 & 0 & $\vdots$ & 0 & 0 \\
\hline & sq7eə $\mathbb{C}$ & 0 & 0 & $\vdots$ & 0 & $\theta$ \\
\hline & squequit & -1 & $N$ & $\vdots$ & $\mapsto$ & 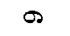 \\
\hline \multirow{6}{*}{ 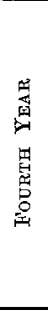 } & 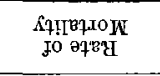 & $\underset{\infty}{\infty}$ & $\S$ & $\vdots$ & 8 & $\underset{T}{E}$ \\
\hline & 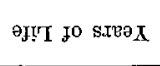 & 0 & $\stackrel{10}{i}$ & $\vdots$ & $\rightarrow$ & $\stackrel{10}{1}$ \\
\hline & 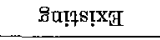 & $\infty$ & 다 & $\vdots$ & 0 & $\infty$ \\
\hline & S[вмварчт!М & 0 & 10 & $\vdots$ & 0 & 20 \\
\hline & sufeəc & $\infty$ & 0 & $\vdots$ & 0 & $n$ \\
\hline & squвxqua & $\theta$ & $\Theta$ & $\vdots$ & $r$ & ถి \\
\hline \multirow{6}{*}{ 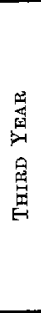 } & 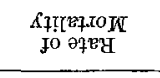 & $\begin{array}{l}8 \\
8\end{array}$ & 8 & 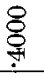 & ᄋ్ & 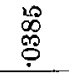 \\
\hline & әر!T Jo sxeə $X$ & $F$ & $\stackrel{\infty}{-1}$ & ติ & 9 & $\stackrel{\leftrightarrow}{N}$ \\
\hline & 8ut?s!x? & 0 & 0 & 0 & 0 & 0 \\
\hline & 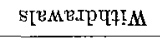 & म & $\ddot{4}$ & $m$ & $r$ & $\stackrel{9}{9}$ \\
\hline & suł8ә & 0 & 0 & - & 0 & -1 \\
\hline & squeriquit & $\stackrel{\infty}{\sim}$ & $\underset{-1}{\pi}$ & $\infty$ & $r$ & $\infty$ \\
\hline \multirow{6}{*}{ 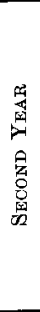 } & 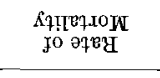 & 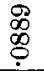 & 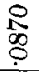 & $\begin{array}{l}8 \\
10 \\
9\end{array}$ & $\begin{array}{l}8 \\
8 \\
\end{array}$ & $\begin{array}{l}\mathscr{8} \\
\stackrel{9}{9}\end{array}$ \\
\hline & 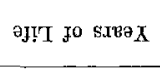 & ஸัง & ติ & チ & -1 & in \\
\hline & 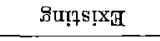 & 0 & $\mathrm{O}$ & 0 & 0 & $\infty$ \\
\hline & \$[вметрчұ!M & 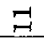 & $\stackrel{N}{N}$ & ov & 0 & 召 \\
\hline & รuาะว 1 & $N$ & N & -1 & 0 & 10 \\
\hline & sque.тuस & $\begin{array}{l}\infty \\
\text { G1 }\end{array}$ & $\mathscr{9}$ & 20 & - & $\mathscr{B}$ \\
\hline \multirow{6}{*}{ 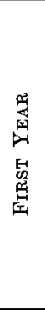 } & 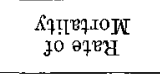 & 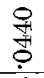 & $\begin{array}{l}\mathbb{N} \\
0 \\
\infty \\
T-1 \\
\end{array}$ & $\begin{array}{l}9 \\
\text { ज़ } \\
\qquad\end{array}$ & $\stackrel{8}{8}$ & 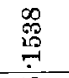 \\
\hline & әЈ!T Jo s.นษ $\boldsymbol{X}$ & $\begin{array}{l}10 \\
19 \\
+7\end{array}$ & 10 & $\stackrel{10}{0}$ & $\stackrel{29}{\rightarrow}$ & $\stackrel{10}{0}$ \\
\hline & öu!qsext & 0 & 0 & 0 & 0 & 0 \\
\hline & รүвм⿻хрчатМ & 9 & 政 & 10 & 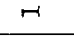 & 5 \\
\hline & sчteea & 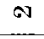 & $Q_{R=1}$ & 10 & 0 & 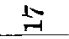 \\
\hline & squextufr & 10 & 0 & $\stackrel{0}{n-1}$ & ज) & 곡 \\
\hline & 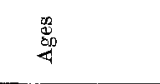 & $\begin{array}{l}\text { : } \\
1 \\
0 \\
0\end{array}$ & $\stackrel{\infty}{\infty}$ & 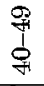 & ఝ̊ & $=8$ \\
\hline
\end{tabular}

